\title{
Rotating periodic solutions for second order systems with Hartman-type nonlinearity
}

\author{
Jian Li ${ }^{1,2}$, Xiaojun Chang ${ }^{1 *}$ and Yong $\mathrm{Li}^{1}$
}

"Correspondence:
changxj1982@hotmail.com
1School of Mathematics and
Statistics \& Center for Mathematics
and Interdisciplinary Sciences,
Northeast Normal University,
Changchun, China
Full list of author information is
available at the end of the article

available at the end of the article

\begin{abstract}
In this paper, by a constructive proof based on the homotopy continuation method, we prove that the second order system $x^{\prime \prime}=g(t, x)$ admits rotating periodic solutions with form $u(t+T)=Q u(t)$ for any orthogonal matrix $Q$ when the nonlinearity $g$ admits the Hartman-type condition.
\end{abstract}

MSC: 34B15; 34C25; 65L99

Keywords: Rotating periodic solutions; Second order systems; Hartman-type nonlinearity; Homotopy continuation method

\section{Introduction and main results}

In this paper we study the following second order system:

$$
x^{\prime \prime}=g(t, x),
$$

where $g$ is continuous from $[0, T] \times \mathbb{R}^{n}$ to $\mathbb{R}^{n}$ and satisfies the following Hartman-type condition:

(H) there exists $\eta>0$ such that

$$
g(t, x) \cdot x \geq 0, \quad \forall t \in[0, T], \forall x \in \mathbb{R} \text { with }|x|=\eta .
$$

In 1960, Hartman [1] firstly studied the existence of system (1.1) with Dirichlet boundary value conditions under the assumption (1.2). In 1971, Knobloch [2] obtained the existence of periodic solutions for (1.1) under the Hartman-type condition $(H)$ when $g$ is locally Lipschitzian in $x$. The result was extended to the case that $g$ is continuous by Rouche and Mawhin [3] in 1973. In [4], Mawhin applied the Schauder fixed point theorem to obtain periodic solutions of $p$-Laplacian ordinary differential systems under assumption $(H)$. In $[5,6]$, some results are obtained on periodic solutions of ordinary differential systems involving singular $\phi$-Laplace operator and $\phi(t)$-Laplace operator under the Hartman-type condition $(H)$. For some variants and extensions, one can see [7-11] and the references therein.

This paper is devoted to showing that, under the Hartman condition $(H)$, the system (1.1) admits the type of solutions with form $u(t+T)=Q u(t)$ for any $Q \in O(n)$ when the

(c) The Author(s) 2018. This article is distributed under the terms of the Creative Commons Attribution 4.0 International License (http://creativecommons.org/licenses/by/4.0/), which permits unrestricted use, distribution, and reproduction in any medium, provided you give appropriate credit to the original author(s) and the source, provide a link to the Creative Commons license, and indicate if changes were made. 
nonlinearity $g$ amidts the corresponding rotating periodic structure. Here $O(n)$ denotes the orthogonal group on $\mathbb{R}^{n}$. Usually, we call the solutions by rotating periodic solutions (or Q-rotating periodic solutions related to the matrix $Q$ ).

Theorem 1.1 Assume that $g \in C\left(\mathbb{R} \times \mathbb{R}^{n}, \mathbb{R}^{n}\right)$ and there exists some $Q \in O(n)$ such that $g(t+T, x)=Q g\left(t, Q^{-1} x\right), \forall t \in \mathbb{R}$. If the assumption (H) holds, then (1.1) has at least one Q-rotating periodic solution.

Example 1.2 Define $g(t, x)=a(t)|x|^{p-2} x, \forall x \in \mathbb{R}^{n}$, where $a \in C(\mathbb{R}, \mathbb{R})$ with $a(t)>0$ and $a(t+$ $T)=a(t)$ for all $t \in \mathbb{R}, p \geq 2$. Clearly, by Theorem 3.1 in [4] we can obtain the existence of at least one periodic solution, while here by Theorem 1.1 we can get infinitely many periodic solutions and quasi-periodic solutions of (1.1) by taking different $Q$. It should be noticed that if $Q \equiv I_{n}$, then the corresponding rotating periodic solution is just a periodic solution of (1.1), while if $Q \not \equiv I_{n}$, we may obtain some existence results on quasi-periodic solutions of (1.1). In fact, we can take $Q$ by

$$
\Lambda_{1} \doteq\left(\begin{array}{lll}
R\left(\theta_{1}\right) & & \\
& \ldots & \\
& & R\left(\theta_{m}\right)
\end{array}\right)
$$

if $n=2 l$ for some $m, l \in \mathbb{Z}^{+}$, or

$$
\Lambda_{2} \doteq\left(\begin{array}{llll}
R\left(\theta_{1}\right) & & & \\
& \cdots & & \\
& & R\left(\theta_{m}\right) & \\
& & & 1
\end{array}\right)
$$

if $n=2 l+1$ for some $m, l \in \mathbb{Z}^{+}$, where $\theta_{i} \in[0,2 \pi], i=1,2, \ldots, m$, and there exist $i \in$ $\{1,2, \ldots, m\}$ such that $\theta_{i}$ is an irrational multiplier of $\frac{\pi}{T}$. Here $R(\theta)=\left(\begin{array}{c}\cos \theta-\sin \theta \\ \sin \theta \\ \cos \theta\end{array}\right)$.

Our proof is based on the homotopy continuation method together with a prior estimate. This method was introduced by Kellogg, Li and Yorke [12] and afterward many authors applied this method to obtain a constructive proof for the existence of periodic solutions of second order ordinary differential, one can see [13-15] and the references. For the periodic solution case, i.e., $Q \equiv I_{n}$, as shown in [15], one can transform the problem into one of finding certain solutions of the Cauchy problem by introducing a homotopy parameter $\lambda \in[0,1]$. Following the $C^{1}$ path of solutions of the Cauchy problem starting from a simpler auxiliary equation corresponding to $\lambda=0$, one can obtain the desired periodic solutions corresponding to $\lambda=1$. However, when $\operatorname{Ker}\left(I_{n}-Q\right) \not \equiv \mathbb{R}^{n}$, it seems difficult to apply this idea to our problem directly. Here we modify this idea and gluing two $C^{1}$ paths of solutions together to obtain a continuous path of solutions, by which we can track along the trajectory to obtain the rotating periodic solutions (specially, quasi-periodic solutions) of (1.1). For some recent work on rotating periodic solutions of ODEs, one can see [16-19] and the references.

Remark In [15], Lu, Li and Su applied the homotopy continuation method to give a constructive proof of Mawhin's continuation theorem in [20], and thus established a global 
method for finding periodic solutions of ordinary differential equations (systems). Our results can be seen as a generalization of the results in [15] from periodic solutions case to the rotating periodic case when $g$ satisfies the Hartman-type nonlinearity.

This paper is organized as follows. In Sect. 2 we give a prior estimate and obtain the proof of Theorem 1.1 by applying the homotopy continuation method. In Sect. 3, some useful facts for the homotopy continuation method are collected. For convenience, we introduce some notations and definitions. $L^{p}(0, T)(1<p \leq \infty)$ denotes the usual Lebesgue measurable space with norm $\|\cdot\|_{p} . C^{m} \doteq C^{m}\left(\mathbb{R}, \mathbb{R}^{n}\right)(m \in \mathbb{N})$ denotes the space of $m$-times continuously differential real functions from $\mathbb{R}$ to $\mathbb{R}^{n}$ with norm

$$
\|u\|_{C^{m}}=\max _{t \in \mathbb{R}}|u(t)|+\max _{t \in \mathbb{R}}\left|u^{\prime}(t)\right|+\cdots+\max _{t \in \mathbb{R}}\left|u^{(m)}(t)\right|
$$

Specially, we denote $\|\cdot\|=\|u\|_{C^{1}}$.

\section{Main results}

Firstly, we consider the following auxiliary problem:

$$
\left\{\begin{array}{l}
x^{\prime \prime}=\lambda\left[x-\phi_{\eta}(x)+g\left(t, \phi_{\eta}(x)\right)\right] \\
x(T)=Q x(0)
\end{array}\right.
$$

where $\lambda \in(0,1]$ and $\phi_{\eta}: \mathbb{R}^{n} \rightarrow \mathbb{R}^{n}$ is defined by

$$
\phi_{\eta}(x)= \begin{cases}x & \text { if }|x| \leq \eta, \\ \eta \frac{x}{|x|} & \text { if }|x|>\eta\end{cases}
$$

where $\eta$ is taken in $(H)$.

Lemma 2.1 Assume that $(\lambda, x)$ is a solution pair of (2.1). Then there exists $\rho>0$ such that $\|x\|<\rho$. Furthermore, if $x$ is a solution of (2.1) with $\lambda=1$, then $x$ is a Q-rotating periodic solution of (1.1).

Proof

Assume that $(\lambda, x)$ is a solution pair of (2.1). Multiplying (2.1) with $x$, by $Q \in O(n)$ we get

$$
\begin{aligned}
& \int_{0}^{T}\left|x^{\prime}(s)\right|^{2} d s+\lambda \int_{0}^{T}|x(s)|^{2} d s \\
& \quad=\lambda \int_{0}^{T} \phi_{\eta}(x) \cdot x(s) d s-\lambda \int_{0}^{T} g\left(s, \phi_{\eta}(x)\right) \cdot x(s) d s \\
& \quad \leq \lambda\left[\left(\int_{0}^{T}\left|\phi_{\eta}(x)\right|^{2} d s\right)^{\frac{1}{2}}+\left(\int_{0}^{T}\left|g\left(s, \phi_{\eta}(x)\right)\right|^{2} d s\right)^{\frac{1}{2}}\right]\left(\int_{0}^{T}|x(s)|^{2} d s\right)^{\frac{1}{2}} .
\end{aligned}
$$

By $\lambda \in(0,1]$, we have

$$
\int_{0}^{T}|x(s)|^{2} d s \leq\left[\left(\int_{0}^{T}\left|\phi_{\eta}(x)\right|^{2} d s\right)^{\frac{1}{2}}+\left(\int_{0}^{T}\left|g\left(s, \phi_{\eta}(x)\right)\right|^{2} d s\right)^{\frac{1}{2}}\right]^{2}
$$


and

$$
\begin{aligned}
\int_{0}^{T}\left|x^{\prime}(s)\right|^{2} d s & \leq\left[\left(\int_{0}^{T}\left|\phi_{\eta}(x)\right|^{2} d s\right)^{\frac{1}{2}}+\left(\int_{0}^{T}\left|g\left(s, \phi_{\eta}(x)\right)\right|^{2} d s\right)^{\frac{1}{2}}\right]\left(\int_{0}^{T}|x(s)|^{2} d s\right)^{\frac{1}{2}} \\
& \leq\left[\left(\int_{0}^{T}\left|\phi_{\eta}(x)\right|^{2} d s\right)^{\frac{1}{2}}+\left(\int_{0}^{T}\left|g\left(s, \phi_{\eta}(x)\right)\right|^{2} d s\right)^{\frac{1}{2}}\right]^{2} .
\end{aligned}
$$

Since $\phi_{\eta}$ is continuous and bounded, it follows that there exists some $C_{1}>0$ independent of $\lambda$ and $x$ such that

$$
\left\|x^{\prime}\right\|_{2}+\|x\|_{2} \leq C_{1}
$$

which implies that there exist $\sigma_{1}, \sigma_{2} \in[0, T]$ such that $\max \left\{\left|x\left(\sigma_{1}\right)\right|,\left|x^{\prime}\left(\sigma_{2}\right)\right|\right\} \leq 2 \frac{C_{1}}{\sqrt{T}}$. Denote $h(t, x)=x-\phi_{\eta}(x)+g\left(t, \phi_{\eta}(x)\right)$. Then

$$
\begin{aligned}
h(t+T, x) & =x-\phi_{\eta}(x)+g\left(t+T, \phi_{\eta}(x)\right) \\
& =Q\left[Q^{-1} x-\phi_{\eta}\left(Q^{-1} x\right)\right]+Q g\left(t+T, Q^{-1} \phi_{\eta}(x)\right) \\
& =Q\left[Q^{-1} x-\phi_{\eta}\left(Q^{-1} x\right)+g\left(t, \phi_{\eta}\left(Q^{-1} x\right)\right)\right] \\
& =Q h\left(t, Q^{-1} x\right) .
\end{aligned}
$$

It is not difficult to see that $x(t+T)=Q x(t)$ for any $t \in \mathbb{R}$. By $Q \in O(n)$ we can see that

$$
|x(t+m T)|=\left|Q^{m} x(t)\right|=|x(t)|, \quad \forall t \in \mathbb{R}, \forall m \in \mathbb{Z}^{+} .
$$

By $x(t)=x\left(\sigma_{1}\right)+\int_{\sigma_{1}}^{t} x^{\prime}(s) d s$ and (2.2) it follows that

$$
\|x\|_{\infty} \leq C_{2}
$$

for some $C_{2}>0$ independent of $\lambda$ and $x$. Integrating (2.1) from $\sigma_{2}$ to $t$, we obtain

$$
x^{\prime}(t)=x^{\prime}\left(\sigma_{2}\right)+\lambda \int_{\sigma_{2}}^{t}\left[x(s)-\phi_{\eta}(x(s))+g\left(t, \phi_{\eta}(x(s))\right)\right] d s .
$$

Similar arguments to above imply that

$$
\left\|x^{\prime}\right\|_{\infty} \leq C_{3}
$$

for some $C_{3}>0$ independent of $\lambda$ and $x$. Denote $\rho=\max \left\{C_{2}, C_{3}, \eta+1\right\}+1$. Then we get $\|x\|<\rho$.

We claim that $|x(t)| \leq \eta$ for all $t \in[0, T]$, i.e., if $x$ is a solution of (2.1) with $\lambda=1$, then $x$ is a $Q$-rotating periodic solution of (1.1). Indeed, for each $t \in[0, T]$ such that $|x(t)|>\eta$, by (2.1) with $\lambda=1$ it follows that

$$
\begin{aligned}
\frac{d}{d t}\left[x^{\prime}(t) \cdot x(t)\right] & =x^{\prime \prime}(t) \cdot x(t)+\left|x^{\prime}(t)\right|^{2} \\
& =\left[|x(t)|^{2}-\phi_{\eta}(x(t)) \cdot x(t)+g\left(t, \phi_{\eta}(x(t))\right) \cdot x(t)\right]+\left|x^{\prime}(t)\right|^{2}
\end{aligned}
$$




$$
\begin{aligned}
& \geq\left[|x(t)|^{2}-\eta \frac{x(t)}{|x(t)|} \cdot x(t)+g\left(t, \eta \frac{x(t)}{|x(t)|}\right) \cdot\left(\eta \frac{x(t)}{|x(t)|} \frac{|x(t)|}{\eta}\right)\right] \\
& \geq|x(t)|(|x(t)|-\eta) \\
& >0 .
\end{aligned}
$$

If $|x(t)|>\eta$ for all $t \in \mathbb{R}$, then (2.7) holds for all $t \in \mathbb{R}$, which is contrary to (2.4). If $\left|x\left(t_{1}\right)\right| \leq \eta$ for some $t_{1} \in[0, T]$ and $\left|x\left(t_{2}\right)\right|>\eta$ for some $t_{2} \in[0, T]$, then we can find $\tau, \xi \in \mathbb{R}$ with $\xi>\tau$ such that

$$
|x(\tau)|=\eta, \quad|x(\xi)|=\max _{t \in \mathbb{R}}|x(t)|>\eta, \quad|x(t)|>\eta, \quad \forall t \in(\tau, \xi) .
$$

Clearly,

$$
\left.\frac{d}{d t}\left[|x(t)|^{2}\right]\right|_{t=\xi}=2 x(\xi) \cdot x^{\prime}(\xi)=0 .
$$

Then, by (2.7) it follows that

$$
\frac{d}{d t}\left[\frac{1}{2}|x(t)|^{2}\right]=x(t) \cdot x^{\prime}(t)<x(\xi) \cdot x^{\prime}(\xi)=0, \quad \forall t \in(\tau, \xi),
$$

which together with (2.8) implies that

$$
\eta^{2}<|x(\xi)|^{2}<|x(\tau)|^{2}=\eta^{2}
$$

This is a contradiction. Hence, $|x(t)| \leq \eta$ for all $t \in \mathbb{R}$. Thus $x$ satisfies $x^{\prime \prime}=g(t, x)$ and $x(t+T)=Q x(t)$, i.e., $x$ is a $Q$-rotating periodic solution of (1.1).

Proof of Theorem 1.1 By $Q \in O(n)$ and the knowledge in linear algebra, there exist an invertible matrix $A$ and an orthogonal matrix $B$ such that $Q=A^{-1} B A$, where $B$ has the form

$$
B=\left(\begin{array}{ll}
I_{\alpha_{1}} & \\
& \hat{Q}
\end{array}\right)
$$

where $\alpha_{1}=\operatorname{dim} \operatorname{Ker}\left(I_{n}-B\right), \hat{Q}$ is a $\beta_{1} \times \beta_{1}$ orthogonal matrix with $\beta_{1}=n-\alpha_{1}$ such that 1 is not an eigenvalue of $\hat{Q}$. Furthermore, we can get

$$
\mathbb{R}^{n}=\operatorname{Ker}\left(I_{n}-B\right) \oplus \operatorname{Im}\left(I_{n}-B\right)
$$

Let $\hat{P}_{1}, \hat{P}_{2}: \mathbb{R}^{n} \rightarrow \mathbb{R}^{n}$ be orthogonal projectors such that $\operatorname{Im} \hat{P}_{1}=\operatorname{Ker}\left(I_{n}-B\right)$ and $\operatorname{Im} \hat{P}_{2}=$ $\operatorname{Im}\left(I_{n}-B\right)$, respectively. Let $B_{r_{1}}^{\alpha_{1}}(0)$ be the open sphere in $\operatorname{Ker}\left(I_{n}-B\right)$ with center 0 and radius $r_{1}>0$, and $B_{r_{2}}^{\beta_{1}}(0)$ be the open sphere in $\operatorname{Im}\left(I_{n}-B\right)$ with center 0 and radius $r_{2}>0$.

In view of $g(t+T, x)=Q g\left(t, Q^{-1} x\right)$, letting $f(t, x)=A g\left(t, A^{-1} x\right)$, we get

$$
\begin{aligned}
f(t+T, x) & =A g\left(t+T, A^{-1} x\right) \\
& =A Q g\left(t, Q^{-1} A^{-1} x\right)
\end{aligned}
$$




$$
\begin{aligned}
& =B A g\left(t, A^{-1} B^{-1} x\right) \\
& =B f\left(t, B^{-1} x\right), \quad \forall x \in \mathbb{R}^{n} .
\end{aligned}
$$

Set $y=A x$. If $x$ is a solution of (2.1), then $y$ satisfies

$$
\left\{\begin{array}{l}
y^{\prime \prime}=\lambda\left[y-\phi_{\eta}(y)+A g\left(t, A^{-1} \phi_{\eta}(y)\right)\right] \doteq \lambda \varphi(t, y), \\
y(T)=B y(0) .
\end{array}\right.
$$

By (2.9) it is clear that $\varphi(t+T, y)=B \varphi\left(t, B^{-1} y\right)$ for all $t \in \mathbb{R}$ and $y \in \mathbb{R}^{n}$.

Case 1: $\operatorname{Ker}\left(I_{n}-B\right) \neq\{0\}$.

Define

$$
H(\xi, \mu)=\mu \xi+(1-\mu) \frac{1}{T} \int_{0}^{T} \hat{P}_{1} \varphi(s, \xi) d s, \quad \forall \xi \in \operatorname{Ker}\left(I_{n}-B\right), \forall \mu \in[0,1] .
$$

For $\xi \in \operatorname{Ker}\left(I_{n}-B\right)$ with $|\xi| \geq \rho$, where $\rho$ is taken from Lemma 2.1, we have

$$
\begin{aligned}
H(\xi, \mu) \cdot \xi & =\mu|\xi|^{2}+(1-\mu) \frac{1}{T} \int_{0}^{T} \hat{P}_{1} \varphi(s, \xi) d s \cdot \xi \\
& =\mu|\xi|^{2}+(1-\mu) \frac{1}{T} \int_{0}^{T}\left[|\xi|^{2}-\eta|\xi|+\hat{P}_{1} g\left(s, \eta \frac{\xi}{|\xi|}\right) \cdot \eta \frac{\xi}{|\xi|} \frac{|\xi|}{\eta}\right] d s \\
& >0 .
\end{aligned}
$$

Then, for any $r \geq \rho$,

$$
\operatorname{deg}_{B}\left(\Phi, B_{r}^{\alpha_{1}}(0), 0\right) \neq 0,
$$

where $\Phi(\xi) \doteq \frac{1}{T} \int_{0}^{T} \hat{P}_{1} \varphi(s, \xi) d s, \forall \xi \in \operatorname{Ker}\left(I_{n}-B\right)$.

Applying the Weierstrass's theorem, we can approximate $\varphi$ by a $C^{2}$ function sequence $\left\{\varphi_{k}\right\}$ such that

$$
\lim _{k \rightarrow+\infty} \varphi_{k}(t, y)=\varphi(t, y)
$$

uniformly in $t \in[0, T]$ and $y \in \overline{B_{\rho}(0)}$. Without loss of generality, we may assume that

$$
\left\|\varphi_{k}-\varphi\right\|_{\infty} \leq \frac{1}{2}, \quad \forall k \in \mathbb{Z}^{+} .
$$

By the continuation and definition of the Brouwer degree, there exists a constant $\sigma_{1}>0$ sufficiently small such that

$$
\operatorname{deg}_{B}\left(\Phi_{k}, B_{\rho}^{\alpha_{1}}(0), d\right) \neq 0
$$

for all $d \in B_{\sigma_{1}}^{\alpha_{1}}(0) \backslash\{0\}$, where $\Phi_{k}(\xi) \doteq \frac{1}{T} \int_{0}^{T} \hat{P}_{1} \varphi_{k}(s, \xi) d s, \forall \xi \in \operatorname{Ker}\left(I_{n}-B\right)$. Then the perturbed boundary value problem

$$
\left\{\begin{array}{l}
y^{\prime \prime}=\lambda \varphi_{k}(t, y)+(1-\lambda) \frac{1}{T} \int_{0}^{T} \hat{P} \varphi_{k}(s, y(s)) d s-(1-\lambda) d, \\
y(T)=B y(0)
\end{array}\right.
$$


has at least one solution $y_{0}(t) \equiv \xi_{0} \in B_{\rho}^{\alpha_{1}}(0)$ for any $d \in B_{\sigma_{1}}^{\alpha_{1}}(0) \backslash\{0\}$ when $\lambda=0$.

For $\lambda \in(0,1]$, denote $y_{k}(t, \xi, \lambda)$ as the solution of $y^{\prime \prime}=\lambda \varphi_{k}(t, y)$ with initial value $y_{k}(0, \xi, \lambda)=\xi$ for $\xi \in \mathbb{R}^{n}$.

By the Sard's theorem, for almost all $d \in B_{\sigma_{1}}^{\alpha_{1}}(0), d$ is a regular value of $\Phi_{k}$. Denote the set of all such points of $d^{\prime}$ s by $\Theta$. Denote $\Lambda_{1} \equiv \Theta \oplus B_{\rho}^{\beta_{1}}(0)$. For $\xi \in \mathbb{R}^{n}$ we can write it as $\xi=\hat{\xi}+\breve{\xi}$, where $\hat{\xi} \in \operatorname{Ker}\left(I_{n}-B\right)$ and $\breve{\xi} \in \operatorname{Im}\left(I_{n}-B\right)$. Define the homotopy map $H_{k}$ : $\Lambda_{1} \times \mathbb{R}^{n} \times[0,1]$ by

$$
H_{k}(d, \xi, \lambda)=\Psi_{k}(\xi, \lambda)-(1-\lambda) d,
$$

where $\Psi_{k}(\xi, \lambda): \mathbb{R}^{n} \times[0,1] \rightarrow \mathbb{R}^{n}$ is defined by

$$
\begin{aligned}
\Psi_{k}(\xi, \lambda)= & \left(\frac{1}{T} \int_{0}^{T} \hat{P}_{1} \varphi_{k}\left(s, y_{k}(s, \xi, \lambda)\right) d s,\right. \\
& \left.\frac{1}{T}\left(I_{n}-B\right) \breve{\xi}+\frac{\lambda}{T} \int_{0}^{T} \hat{P}_{2} \varphi_{k}\left(s, y_{k}(s, \xi, \lambda)\right) d s\right) .
\end{aligned}
$$

By (2.14), for any $d \in \Theta$, it follows that

$$
\begin{aligned}
& \operatorname{deg}_{B}\left(H_{k}(d, \cdot, 0), B_{\rho}^{\alpha_{1}}(0) \oplus B_{\rho}^{\beta_{1}}(0), d\right) \\
& \quad=\operatorname{deg}_{B}\left(\Phi_{k}, B_{\rho}^{\alpha_{1}}(0), d\right) \cdot \operatorname{deg}_{B}\left(\frac{1}{T}\left(I_{n}-B\right) \breve{\xi}, B_{\rho}^{\beta_{1}}(0), 0\right) \neq 0 .
\end{aligned}
$$

It is easily seen that there exists $\lambda_{0}>0$ small enough such that, for all $d \in \Theta, 0$ is a regular value of $H_{k}(d, \xi, \lambda)$ for any $\lambda \in\left[0, \lambda_{0}\right)$. Denote the set of all such points of $d^{\prime}$ s by $\Lambda_{1}^{*}$. For any $d \in \Lambda_{1}^{*}$, denote $H_{k, d}(\xi, \lambda)=H_{k}(d, \xi, \lambda)$ for all $(\xi, \lambda) \in \mathbb{R}^{n} \times[0,1)$. Applying the parametrized Sard theorem, we know that for almost all the points $d \in \Lambda_{1}^{*}, 0$ is a regular value of $\left.H_{k, d}(\xi, \lambda)\right|_{\mathbb{R}^{n} \times\left(0, \lambda_{0}\right)}$. Denote the set of all such points of $d^{\prime}$ s as $\Lambda_{2}^{*}$. Clearly, for any $d \in \Lambda_{2}^{*}, 0$ is a regular value of $\left.H_{k, d}(\xi, \lambda)\right|_{\mathbb{R}^{n} \times\left[0, \lambda_{0}\right)}$. Then, by Lemma 3.3, for every $d \in \Lambda_{2}^{*}$, there exists a $C^{1}$ curve $(\xi(s), \lambda(s))(s \geq 0)$ such that

$$
\begin{aligned}
& H_{k, d}(\xi(s), \lambda(s))=0, \quad 0 \leq \lambda<\lambda_{0}, \\
& (\xi(0), \lambda(0))=\left(\xi_{0}, 0\right) .
\end{aligned}
$$

By Lemma 3.5 we can see that $(\xi(s), \lambda(s))$ satisfies the following differential equation:

$$
\left\{\begin{array}{l}
\frac{d \xi_{i}}{d s}(s)=(-1)^{i+1} \operatorname{det} H_{k, d, i}^{\prime}, \quad i=1, \ldots, n \\
\frac{d \lambda}{d s}(s)=(-1)^{n+2} \operatorname{det} H_{k, d, n+1}^{\prime}, \\
\xi(0), \lambda(0))=\left(\xi_{0}, 0\right) .
\end{array}\right.
$$

Now we show that the curve $(\xi(s), \lambda(s))$ is not a loop. In fact, since $\overline{B_{\rho}^{\alpha_{1}}(0)}$ is compact, it is not difficult to see that the number of such $\xi_{0}$ is finite, without loss of generality, we denote them as $\xi_{01}, \xi_{02}, \ldots, \xi_{0 m}$. Let $\left(\xi\left(s, \xi_{0 i}\right), \lambda\left(s, \xi_{0 i}\right)\right)$ be a path of the homotopy $H_{k, d}(\xi(s), \lambda(s))$ with $(\xi(0), \lambda(0))=\left(\xi_{0 i}, 0\right)$. When $\lambda=0$ and $d \in \Lambda_{2}^{*}$, denoting $H_{k, d, \xi}^{\prime}\left(\xi_{0 i}\right)=\left.\frac{\partial H_{k, d}}{\partial \xi}\right|_{\xi=\xi_{0 i}}$, by the fact that 0 is a regular value of $\left.H_{k, d}(\xi, \lambda)\right|_{\mathbb{R}^{n} \times\left[0, \lambda_{0}\right)}$ for any $d \in \Lambda_{2}^{*}$ it follows that 
$\operatorname{det} H_{k, d, \xi}^{\prime}\left(\xi_{0 i}\right) \neq 0$ for $i=1,2, \ldots, m$. By the implicit function theorem we can see that the conclusion follows.

Using Lemma 3.4 it follows that each curve $\left(\xi\left(s, \xi_{0 i}\right), \lambda\left(s, \xi_{0 i}\right)\right)(i \in\{1,2, \ldots, m\})$ is an interval, which maybe open, closed or semi-closed. We claim there exists some $i_{0} \in$ $\{1,2, \ldots, m\}$ such that the curve $\left(\xi\left(s, \xi_{0 i_{0}}\right), \lambda\left(s, \xi_{0 i_{0}}\right)\right)$ starts from $\overline{B_{\rho}^{\alpha_{1}}(0)} \times\{0\}$ and doesn't end on $\overline{B_{\rho}(0)} \times\{0\}$. Indeed, if not, then $m=2 l$ for some $l \in \mathbb{Z}^{+}$. Without loss of generality, we may assume that $\left(\xi\left(s, \xi_{0 i}\right), \lambda\left(s, \xi_{0 i}\right)\right)$ starts from $\left(\xi_{0 i}, 0\right) \in \overline{B_{\rho}^{\alpha_{1}}(0)} \times\{0\}$ and ends at $\left(\xi_{0(l+i)}, 0\right) \in \overline{B_{\rho}(0)} \times\{0\}$ for all $i=1,2, \ldots, l$. By Lemma 3.6 we can get that

$$
\operatorname{sign} \operatorname{det} H_{k, d, \xi}^{\prime}\left(\xi_{0 i}\right)=-\operatorname{sign} \operatorname{det} H_{k, d, \xi}^{\prime}\left(\xi_{0(l+i)}\right), \quad i=1,2, \ldots, l .
$$

Thus by the continuation and definition of the Brouwer degree it follows that, for $d \in \Theta$,

$$
\begin{aligned}
& \operatorname{deg}_{B}\left(H_{k}(d, \cdot, 0), B_{\rho}^{\alpha_{1}}(0) \oplus B_{\rho}^{\beta_{1}}(0), d\right) \\
& \quad=\operatorname{deg}_{B}\left(H_{k, d}(\cdot, 0), B_{\rho}^{\alpha_{1}}(0) \oplus B_{\rho}^{\beta_{1}}(0), 0\right)=\sum_{i=1}^{2 l} \operatorname{sign} \operatorname{det} H_{k, d, \xi}^{\prime}\left(\xi_{0 i}\right)=0 .
\end{aligned}
$$

However, this is contrary to (2.16).

Note that $\xi_{0} \in \overline{B_{\rho}^{\alpha_{1}}(0)}$, we can see that there exists $\lambda^{*} \in\left(0, \lambda_{0}\right)$ small enough such that the curve $\xi(s)$ will stay in $\overline{B_{\rho}^{\alpha_{1}}(0)}$ when $0<\lambda(s) \leq \lambda^{*}$. Denote the position $\xi\left(s^{*}\right)$ by $\xi^{*}$ such that $\lambda^{*}=\lambda\left(s^{*}\right)$ for some $s^{*}>0$. Clearly, by the homotopy invariance of Brouwer degree and (2.16), we get

$$
\begin{aligned}
& \operatorname{deg}_{B}\left(H_{k, d}\left(\cdot, \lambda^{*}\right), B_{\rho}^{\alpha_{1}}(0) \oplus B_{\rho}^{\beta_{1}}(0), d\right) \\
& \quad=\operatorname{deg}_{B}\left(H_{k, d}(\cdot, 0), B_{\rho}^{\alpha_{1}}(0) \oplus B_{\rho}^{\beta_{1}}(0), 0\right) \neq 0, \quad \forall d \in \Lambda_{2}^{*} .
\end{aligned}
$$

For any $\lambda \in\left[\lambda^{*}, 1\right)$ and $\xi \in \mathbb{R}^{n}$, since $\left(\frac{\partial H_{k}}{\partial d}, \frac{\partial H_{k}}{\partial \xi}, \frac{\partial H_{k}}{\partial \lambda}\right)=\left(-(1-\lambda) I_{n}, \frac{\partial H_{k}}{\partial \xi}, \frac{\partial H_{k}}{\partial \lambda}\right)$, we can see that, for all $d \in \Lambda_{2}^{*}, 0$ is a regular value of $H_{k, d}(\xi, \lambda)$ for any $\lambda \in\left[\lambda^{*}, 1\right)$. Denote the set of all such points of $d^{\prime}$ s by $\Gamma_{1}^{*}$. Applying the parametrized Sard theorem, we know that for almost all the points $d \in \Gamma_{1}^{*}, 0$ is a regular value of $\left.H_{k, d}(\xi, \lambda)\right|_{\mathbb{R}^{n} \times\left[\lambda^{*}, 1\right)}$. Denote the set of all such points of $d^{\prime}$ s as $\Gamma_{2}^{*}$. Clearly, for any $d \in \Gamma_{2}^{*}, 0$ is a regular value of $\left.H_{k, d}(\xi, \lambda)\right|_{\mathbb{R}^{n} \times[\lambda *, 1)}$. Then, by Lemma 3.3 , for every $d \in \Gamma_{2}^{*}$, there exists a $C^{1}$ curve $(\tilde{\xi}(s), \tilde{\lambda}(s))$, such that

$$
\begin{aligned}
& H_{k, d}(\tilde{\xi}(s), \tilde{\lambda}(s))=0, \quad \lambda^{*} \leq \tilde{\lambda}<1, \\
& (\tilde{\xi}(0), \tilde{\lambda}(0))=\left(\xi^{*}, \lambda^{*}\right) .
\end{aligned}
$$

By Lemma 3.5 we can see that $(\tilde{\xi}(s), \tilde{\lambda}(s))$ satisfies the following differential equation:

$$
\left\{\begin{array}{l}
\frac{d \tilde{\xi}_{i}}{d s}(s)=(-1)^{i+1} \operatorname{det} H_{k, d, i}^{\prime}, \quad i=1, \ldots, n, \\
\frac{d \tilde{\lambda}}{d s}(s)=(-1)^{n+2} \operatorname{det} H_{k, d, n+1}^{\prime}, \\
(\tilde{\xi}(0), \tilde{\lambda}(0))=\left(\xi^{*}, \lambda^{*}\right) .
\end{array}\right.
$$

Since $\varphi_{k}$ is of $C^{2}$ and $\left(\xi^{*}, \lambda^{*}\right) \in B_{\rho}(0) \times\left[\lambda^{*}, 1\right)$, it follows that Cauchy problem (2.19) has the maximal solution $(\tilde{\xi}(s), \tilde{\lambda}(s))_{s \in[0, \tau)}$ in $\overline{B_{\rho}(0)} \times\left[\lambda^{*}, 1\right)$. In what follows, we shall proceed 
to prove that the path tends to $\overline{B_{\rho}(0)} \times\{1\}$. By similar arguments as above, together with the implicit function theorem and (2.18), we get:

(a) The path $(\tilde{\xi}(s), \tilde{\lambda}(s))_{s \in[0, \tau)}$ is not a loop.

(b) The path $(\tilde{\xi}(s), \tilde{\lambda}(s))_{s \in[0, \tau)}$ will not return to some point $\left(\xi_{1}, \lambda^{*}\right)$.

Furthermore, by the above claim it follows that the path $(\tilde{\xi}(s), \tilde{\lambda}(s))_{s \in[0, \tau)}$ will not touch the boundary $\partial B_{\rho}(0) \times\left[\lambda^{*}, 1\right)$. By the idea of the homotopy continuation method (see $[15,21])$, we can obtain that there exists $j^{*} \in\{1,2, \ldots, m\}$ such that $\lambda_{\max , j^{*}}=\sup \left\{\lambda\left(s, \xi_{0 j^{*}}\right)\right.$ : $s \in[0, \tau)\}=1$. Hence $H_{k}\left(d, \xi_{0, j^{*}}, 1\right)=0$ and $y_{k}\left(t, \xi_{0, j^{*}}, 1\right)$ is a solution of $(2.1)$ with $\lambda=1$.

Assume that $\left\{y_{k}\right\}$ is a solution sequence of $(2.10)$ with $\lambda=1$ corresponding to $\left\{\varphi_{k}\right\}$. Firstly, it is easily seen that $\left\{y_{k}\right\} \subset \Omega$ and thus is uniformly bounded. Secondly, to show $\left\{y_{k}\right\}$ is equicontinuous on $[0, T]$, we can obtain by (2.12)-(2.13) and the smooth of $\varphi_{k}$ that

$$
\begin{aligned}
& \left|\varphi_{k}\left(t, y_{k}\right)-\varphi\left(t, y_{k}\right)\right| \leq \frac{1}{2}, \\
& \left|\varphi\left(t, y_{k}\right)\right| \leq M,
\end{aligned}
$$

which implies $\left|\varphi_{k}\left(t, y_{k}\right)\right| \leq M+\frac{1}{2}, \forall k \in \mathbb{Z}^{+}$. By (2.10) with $\lambda=1$ corresponding to $\left\{\varphi_{k}\right\}$ we get $\left|y_{k}^{\prime \prime}\right| \leq M+\frac{1}{2}$. Then $\left\{y_{k}\right\}$ and $\left\{y_{k}^{\prime}\right\}$ are uniformly bounded and equicontinuous on $[0, T]$. By the Arzela-Ascoli theorem it follows that there is a uniformly convergent subsequence of $\left\{y_{k}\right\}$ on $[0, T]$, which is still denoted by $\left\{y_{k}\right\}$, such that

$$
\lim _{k \rightarrow+\infty} y_{k}(t)=y^{*}(t), \quad \lim _{k \rightarrow+\infty} y_{k}^{\prime}(t)=\left(y^{*}\right)^{\prime}(t)
$$

Hence $\lim _{k \rightarrow+\infty} \varphi_{k}\left(t, y_{k}(t)\right)=\varphi\left(t, y^{*}(t)\right)$ and we can easily verify that $x^{*}=A y^{*}$ is a solution of (2.1) with $\lambda=1$.

Case 2: $\operatorname{Ker}\left(I_{n}-B\right)=\{0\}$.

In this case $\alpha_{1}=0$. By approximating $\varphi$ by a $C^{2}$ function sequence $\left\{\varphi_{k}\right\}$, we can consider the homotopy map

$$
\tilde{H}_{k}(d, \xi, \lambda)=\tilde{\Psi}_{k}(\xi, \lambda)-(1-\lambda) d
$$

where $\tilde{\Psi}_{k}(\xi, \lambda): \mathbb{R}^{n} \times[0,1] \rightarrow \mathbb{R}^{n}$ is defined by

$$
\tilde{\Psi}_{k}(\xi, \lambda)=\frac{1}{T}\left(I_{n}-B\right) \xi+\frac{\lambda}{T} \int_{0}^{T} \varphi_{k}\left(s, y_{k}(s, \xi, \lambda)\right) d s
$$

By similar arguments as above we can obtain the existence of $Q$-rotating periodic solutions of (1.1). This completes the proof.

\section{Appendix}

In this section, we collect some basic results on the homotopy continuation method.

Lemma 3.1 ([22] Sard's theorem) If $\Phi: U \subset \mathbb{R}^{m} \rightarrow \mathbb{R}^{n}$ is a $C^{k}$ map on an open set $U$ with $k>\max \{0, m-n\}$, then the set of singular values of $\Phi$ has $n$-dimensional Lebesgue measure zero. Consequently, the set of regular values of $\Phi$ is dense in $\mathbb{R}^{n}$. 
Lemma 3.2 ([23] Parametrized Sard's theorem) Let $V \subset \mathbb{R}^{n}, U \subset \mathbb{R}^{m}$ be open sets and $\Phi: V \times U \rightarrow \mathbb{R}^{k}$ a $C^{r}$ map, where $r>\max \{0, m-k\}$. If $0 \in \mathbb{R}^{k}$ is a regular value of $\Phi$, then for almost all $a \in V, 0$ is a regular value of $\Phi_{a}=\Phi(a, \cdot)$.

Lemma 3.3 ([22]) Let $\Phi: R^{n+1} \rightarrow R^{n}$ be a $C^{1}$ map and 0 a regular value of $\Phi$. Then $\Phi^{-1}(0)$ is a $C^{1}$-manifold of dimension 1 .

Lemma 3.4 ([22]) A $C^{1}$-manifold of dimension 1 is homeomorphic to a loop or an interval (open, closed, or semiclosed).

Lemma 3.5 ([21]) Let the homotopy $\Phi: R^{n+1} \rightarrow R^{n}$ be a $C^{1}$ map and 0 be regular value of $\Phi$. Then each solution $x(s)$ of the Cauchy problem

$$
\frac{d x_{i}}{d s}=(-1)^{i+1} \operatorname{det} \Phi_{i}^{\prime}, \quad x_{i}(0)=x_{i_{0}} \quad(i=1, \ldots, n+1)
$$

is a $C^{1}$ path of $\Phi^{-1}(0)$, where s is a parameter and

$$
\Phi_{i}^{\prime}=\left(\Phi_{x_{1}}, \ldots, \Phi_{x_{i-1}}, \Phi_{x_{i+1}}, \ldots, \Phi_{x_{n+1}}\right) .
$$

Lemma 3.6 ([21]) Let $V \subset R^{n}$ be an open bounded set, $H: V \times[0,1] \rightarrow R^{n}$ a $C^{1}$ homotopy, 0 a regular value of $H$, and the Jacobi matrices $H_{p}^{\prime}(p, 0)\left(p \in H_{0}^{-1}(0)\right), H_{p}^{\prime}(p, 1)\left(p \in H_{0}^{-1}(0)\right)$ be nonsingular, where

$$
H_{0}=H(\cdot, 0), \quad H_{1}=H(\cdot, 1) .
$$

If a path solution $(p(s), \lambda(s))$ connects point $\left(p_{0}, 0\right)$ with $\left(p_{1}, 0\right)$, then

$$
\operatorname{sgn} \operatorname{det} H_{\lambda}^{\prime}\left(P_{0}, 0\right)=-\operatorname{sgn} \operatorname{det} H_{\lambda}^{\prime}\left(P_{1}, 0\right) .
$$

If a path solution $(p(s), \lambda(s))$ of $H$ connects point $\left(p_{0}, 0\right)$ with $\left(p_{1}, 1\right)$, then

$$
\operatorname{sgn} \operatorname{det} H_{\lambda}^{\prime}\left(P_{0}, 0\right)=\operatorname{sgn} \operatorname{det} H_{\lambda}^{\prime}\left(P_{1}, 0\right) .
$$

\section{Conclusions}

In this paper, by a constructive proof based on the homotopy continuation method, we prove that the second order system $x^{\prime \prime}=g(t, x)$ admits rotating periodic solutions with form $u(t+T)=Q u(t)$ for any orthogonal matrix $Q$ when the nonlinearity $g$ admits the Hartman-type condition. In [3], Rouche and Mawhin obtained the existence of periodic solutions of second order system under the Hartman-type condition. In [15], Lu, Li and $\mathrm{Su}$ applied the homotopy continuation method to give a constructive proof of Mawhin's continuation theorem in [20], which can be used to obtain the periodic solutions by track along a $C^{1}$ trajectory. However, when $\operatorname{Ker}\left(I_{n}-Q\right) \not \equiv \mathbb{R}^{n}$, it seems difficult to apply this idea to our problem directly. Here we modify this idea and gluing two $C^{1}$ paths of solutions together to obtain a continuous path of solutions, by which we can obtain the rotating periodic solutions (specially, quasi-periodic periodic solutions) of (1.1). Our results can be seen as a generalization of the results in [15] from periodic solutions case to the rotating periodic case when $g$ satisfies the Hartman-type nonlinearity. 


\section{Funding}

J. Li is supported by NSFC (No. 41601454). X.J. Chang is supported by NSFC (No. 11471067, 11101178), the Fundamental Research Funds for the Central Universities (No. 2412017BJ001) and the Scientific Research Projects of the Education Department of Jilin Province. Y. Li is supported by 973 Program (No. 2013CB834102) and NSFC (No. 11571065, 11171132).

\section{Competing interests}

The authors declare that they have no competing interests.

\section{Authors' contributions}

All authors read and approved the final manuscript.

\section{Author details}

${ }^{1}$ School of Mathematics and Statistics \& Center for Mathematics and Interdisciplinary Sciences, Northeast Normal University, Changchun, China. ${ }^{2}$ College of Information Technology, Jilin Agricultural University, Changchun, China.

\section{Publisher's Note}

Springer Nature remains neutral with regard to jurisdictional claims in published maps and institutional affiliations.

Received: 4 September 2017 Accepted: 9 March 2018 Published online: 21 March 2018

\section{References}

1. Hartman, P.: On boundary value problems for systems of ordinary, nonlinear, second order differential equations. Trans. Am. Math. Soc. 96, 493-509 (1960)

2. Knobloch, H.W.: On the existence of periodic solutions for second order vector differential equations. J. Differ. Equ. 9, 67-85 (1971)

3. Rouche, N., Mawhin, J.: Equations Différentielles Ordinaires, vol. 2. Masson, Paris (1973). English translation Ordinary Differential Equations, Stability and Periodic Solutions, Pitman, Boston (1980)

4. Mawhin, J.: Some boundary value problems for Hartman-type perturbations of the ordinary vector $p$-Laplacian. Nonlinear Anal. 40, 497-503 (2000)

5. Fan, X.L., Wu, H.Q., Wang, F.Z.: Hartman-type results for p(t)-Laplacian systems. Nonlinear Anal. 52, 585-594 (2003)

6. Liu, Q.H., Qian, D.B., Chu, B.: Nonlinear systems with singular vector $\phi$-Laplacian under the Hartman-type condition. Nonlinear Anal. 74, 2880-2886 (2011)

7. Amster, P., Haddad, J.: A Hartman-Nagumo type condition for a class of contractible domains. Topol. Methods Nonlinear Anal. 41, 287-304 (2013)

8. Andres, J., Malaguti, L., Pavlačková, M.: Hartman-type conditions for multivalued Dirichlet problems in abstract spaces. In: Discr. Cont. Dyn. Syst., 10th AIMS Conf. Suppl, pp. 103-111 (2015)

9. Bebernes, J., Schmitt, K.: Periodic boundary value problems for systems of second order differential equations. J. Differ. Equ. 13, 32-47 (1973)

10. Mawhin, J.: Boundary value problems for nonlinear second-order vector differential equations. J. Differ. Equ. 16 257-269 (1974)

11. Schmitt, K.: Periodic solutions of systems of second-order differential equations. J. Differ. Equ. 11, 180-192 (1972)

12. Kellogg, R.B., Li, T.Y., Yorke, J.A.: A constructive proof of the Brouwer fixed-point theorem and computational results. SIAM J. Numer. Anal. 13,473-483 (1976)

13. Li, Y., Lu, X.G., Su, Y.: A homotopy method of finding periodic solutions for ordinary differential equations from the upper and lower solutions. Nonlinear Anal. 24, 1027-1038 (1995)

14. Li, Y., LV, X.R.: Continuation theorems for boundary value problems. J. Math. Anal. Appl. 190, 32-49 (1995)

15. Lu, X.G., Li, Y., Su, Y.: Finding periodic solutions of ordinary differential equations via homotopy method. Appl. Math. Comput. 62, 153-170 (1994)

16. Chang, X.J., Li, Y.: Rotating periodic solutions of second order dissipative dynamical systems. Discrete Contin. Dyn. Syst. 36, 643-652 (2016)

17. Chang, X.J., Li, Y.: Rotating periodic solutions for second-order dynamical systems with singularities of repulsive type. Math. Methods Appl. Sci. 40, 3092-3099 (2017)

18. Liu, G.G., Yang, X., Li, Y.: Rotating periodic solutions for asymptotically linear second-order Hamiltonian systems with resonance at infinity. Math. Methods Appl. Sci. 40, 7139-7150 (2017)

19. Wang, H.R., Yang, X., Li, Y., Li, X.Y.: LaSalle type stationary oscillation theorems for affine-periodic systems. Discrete Contin. Dyn. Syst., Ser. B 22, 2907-2921 (2017)

20. Mawhin, J.: Topological Degree Methods in Nonlinear Boundary Value Problems. CBMS Regional Conference Series in Mathematics, vol. 40. American Mathematical Society, Providence (1979)

21. Carcia, C.B., Zangwill, W.I.: An approach to homotopy and degree theory. Math. Oper. Res. 4, 390-405 (1979)

22. Milnor, J.W.: Topology from the Differentiable Viewpoint. Princeton Landmarks in Mathematics. Princeton University Press, Princeton (1997)

23. Chow, S.N., Mallet, J.P., Yorke, J.A.: Finding zeroes of maps: homotopy methods that are constructive with probability one. Math. Comput. 32,887-899 (1978) 